\title{
ORT.27 - Adalimumab protection trough patents of invention: a comparison of the strategies adopted by the American, Brazilian and European Patent Offices
}

\author{
Marcus Vinicius Paschoal da Silva ${ }^{1 *}$; Iolanda Margherita Fierro ${ }^{1}$. \\ 1INPI - Instituto Nacional da Propriedade Industrial.
}

Introduction: Biological drugs displayed an exponential development in recent years. Monoclonal antibodies (mAbs), in particular, have proved to be one of the main categories of these drugs, incorporating several innovations in their production. In order to protect these innovative products, patent protection has been widely used by companies, from startups to multinationals. However, although allowing the return of investments in the R\&D of new drugs, patent protection creates an exploitation monopoly which leads to an increase of costs by national health systems. According to the Brazilian Ministry of Health, biological drugs represent $40 \%$ of the costs with drugs, but only $2 \%$ of the total drugs volume. These high costs may be due to the exceptions admitted by the sole paragraph of article 40 of the Brazilian Patent Statute, which allows the extension of the patent term if the examination takes more than 10 years, and to the Brazilian Patent Office delay in finishing the examination, the socalled backlog. Therefore, the identification of common points in patent examination among several patent offices could reduce the examination time and, ultimately, the backlog.

Objective: The present study seeks to compare the main arguments pointed out by the American, Brazilian and European Patent Offices (USPTO, BRPTO and EPO, respectively) during the examination of adalimumab patent applications, and the strategies used by the applicants to overcome these objections, in order to retrieve any common points.

Methodology: Adalimumab was selected according to three criteria (i) it is one of the mAb listed with the biggest sales worldwide; (ii) it has patent applications in the American, Brazilian and European Offices; and (iii) it has a complete examination and a published decision in the BRPTO, EPO and USPTO. The applications were searched in the BRPTO database using the keywords antibodies, monoclonal and immunoglobulin in combination with International Patent Classifications (IPC) A61K, C07K and C12N. The American and European counterparts were searched in their respective databases.

Results: Brazil requires some observations in the context of the claims that are not the practice of European or American Offices, such as the insertion of the expression "characterized by". On the other hand, several points in common in the analysis were observed, such as the concern to define the antibody or its CDRs by the specific amino acid sequence (SEQ IDs) and to seclude the antibody described in the invention from a naturally produced one.

Conclusion: Although each Patent Office has its own interpretation on fields such as therapeutic and diagnostic methods, several arguments presented are uniform in the three offices, and an appropriate amending of the claim chart previously to the examination request in Brazil could shorten the examination period and, in last instance, the backlog.

Keywords: Patents; Antibodies; Adalimumab 